\title{
Simulation of a D-T Neutron Source for Neutron Scattering Experiments
}

\author{
T.P. Lou ${ }^{a, b, *}$, B.A. Ludewigt ${ }^{a}$, J.L. Vujic ${ }^{b}$, K.-N. Leung ${ }^{a, b}$ \\ ${ }^{a}$ Accelerator \&f Fusion Research Division, Lawrence Berkeley National Laboratory \\ ${ }^{\mathrm{b}}$ Department of Nuclear Engineering, University of California, Berkeley
}

\begin{abstract}
A new generation of high-yield fusion-based neutron generators is being developed at Lawrence Berkeley National Laboratory. Novel design features provide the potential for neutron yields as high as $10^{14} \mathrm{n} / \mathrm{s}$ in a compact geometry. Such high neutron yields make the neutron generator applicable to the production of thermal or cold neutrons for neutron scattering experiments. This paper describes a scheme to simulate a neutron scattering setup and to estimate neutron flux and resolution with the particle transport code MCNP. For an estimated resolution of $1 \%$ to $4 \%$, depending on the scattered angle and neutron energy, a thermal neutron flux at the sample position of $7 \times 10^{5} \mathrm{n} / \mathrm{cm}^{2} \mathrm{~s}$ has been estimated. Such a neutron generator is particularly suitable for cold neutron scattering experiments due to relatively longer flight times.
\end{abstract}

Key words: neutron scattering, resolution, Monte Carlo, neutron moderation PACS: 28.20.Cz, 28.20.Gd, 29.25.Dz, 61.12.- $\mathrm{q}$ 


\section{Introduction}

Neutrons like other particles exhibit wave properties. According to Bragg's Law, the inter-atomic spacing in condensed matter can be determined by the constructive interference when the path difference of two beams of particles with the same wavelength equals some integer multiple of its wavelength.[1] Because thermal and cold neutrons have wavelengths close to the inter-atomic spacings of condensed matters, they can be used as a probe for these materials. Neutrons also have an advantage of being neutral so they interact with the nucleus rather than the electron cloud. Therefore, neutrons are highly penetrating and sensitive to light atoms. As a result, neutron scattering is a powerful technique that supports many areas of condensed-matter science. It helps scientists to understand the microscopic properties of polymers, proteins, alloys, amorphous materials, liquid crystals, surfactants, magnets and superconductors.

Reactors and spallation neutron sources are commonly used to produce neutrons for neutron scattering experiments. However, many research reactors have been shutdown and decommissioned and only a small number of reactors among those available today provide a neutron flux high enough for neutron scattering experiments.[2] Spallation neutron sources have taken the lead for neutron science. The advantage of a spallation neutron source is its high neutron yield and its pulsing capability. Its pulsing capability enables the usage of most thermal and cold neutrons by the time-of-flight (ToF) technique.[3]

\footnotetext{
* Corresponding author. Address: 1 Cyclotron Road Mail Stop 5R0121, Berkeley, CA 94720-8232; Tel.: +1(510)495-2792; fax: +1(510)486-5105.

Email address: lou@nuc.berkeley.edu (T.P. Lou).
} 
The Spallation Neutron Source (SNS) is a multi-laboratory project which cost more than one billion dollars and must grow a substantial user community. A low cost D-T neutron source suitable for neutron scattering experiments at universities and individual institutes can potentially provide easy access to neutron scattering and foster education of neutron science at all levels. This study investigates the feasibility of using a high yield D-T neutron generator for neutron scattering experiments as an educational and research tool.

The D-T neutron generator described in this study is currently under development and a prototype neutron generator based on the $\mathrm{D}-\mathrm{D}$ fusion reaction is being tested at Lawrence Berkeley National Laboratory (LBNL).[4] This study assumes a D-T neutron generator design with a neutron yield of $10^{14}$ $\mathrm{n} / \mathrm{s}$. Although the neutron yield of a typical spallation neutron source is two orders of magnitude higher than such a D-T neutron generator, a D-T neutron generator source has the advantage of a more compact geometry and lower primary neutron energy. The energy spectrum of source neutrons produced in spallation reactions has a long high-energy (20 MeV to $\mathrm{GeV})$ tail at the forward direction.[5] A sample is not placed in a direct line of sight to the proton beam line in order not to be irradiated by these high energy neutrons. Rather, neutrons are extracted at $90^{\circ}$ to the proton beam line although the highest double differential cross section for the spallation reaction is at $0^{\circ}$.[6] In this work, the potential advantages of a $\mathrm{D}-\mathrm{T}$ neutron generator source for neutron scattering have been studied. Computer simulations have been performed to investigate whether such a system can produce a neutron flux high enough for neutron scattering experiments at useful resolution.

A Monte Carlo code MCNP has been used for the neutron transport problem in this study.[7] MCNP is capable of simulating neutrons in a general 
geometry. However, it does not take crystal lattice orientation into account in its calculation. Software for designing neutron scattering instrumentation (e.g. VITESS) is often used to calculate the resolution by taking an input of neutron flux and pulse shape defined by the user. Parameters like wavelength, divergence and time at the moderator are determined at random. [8] The neutron flux and pulse shape can be determined by a neutron transport code like MCNP or measured experimentally. Then, the calculated or measured values can be fed into a neutron scattering instrumentation code such as VITESS. However, the approach of running instrumentation code with MCNP results is too time--consuming and inefficient if we want a rough estimate of the reso-

lution and a more accurate evaluation of neutron flux at the sample position. Therefore, in this work we developed an MCNP-based scheme to estimate the resolution without using any instrumentation code such as VITESS. A detailed error analysis of this scheme is presented on this paper.

This paper is organized in the following way. In Section 2, the definitions of terms are clarified and an error analysis for the scheme of evaluating the resolution is discussed. In Section 3, the simulation results are presented and the variance reduction method used in our MCNP simulations is discussed. The conclusions are given in Section 4.

\section{Design parameters and error analysis of the adopted scheme}

Knowledge of neutron flux alone is not adequate to evaluate the source performance for a ToF system because the length of the neutron flight path and the moderated neutron pulse shape determine the resolution of the system. According to Bragg's Law, the regular array of atoms in a crystal acts as a 
three-dimensional diffraction grating for any particle exhibitting wave properties. The inter-atomic spacing, $d$, is related to a particular neutron wavelength by:

$$
n \lambda=2 d \sin \theta
$$

where $\mathrm{n}$ is an integer, $\lambda$ is the wavelength of incident neutron, and $\theta$ is an angle that the incident beam makes with one of the planes of atoms (see Fig. 1). Thermal and cold neutrons have wavelengths ranging from 1 to $30 \AA$. [9] An energy range has to be defined in order to calculate the neutron flux. Because thermal and cold neutron sources are associated with different energy ranges, we use the definitions listed in Table 1 in this paper.[9] Applied to ToF techniques, Bragg's Law can be re-written as follows:

$$
d=\frac{n h t}{2 m L \sin \theta} \quad \text { with } \quad \lambda=\frac{h t}{m L}
$$

where $L$ is the total length of the flight path from moderator to sample to detector, $t$ is the neutron flight time, $m$ is the neutron mass, and $h$ is Planck's constant. When a time-dependent problem is simulated in MCNP, the neutron flight time is related to wavelength and, therefore, via Bragg's Law to the inter-atomic spacing for a given scattered angle. Figure 2 is a schematic diagram showing the relationship between the detector panel and sample position. The detector tubes in Fig. 2 are aligned into the page. In our simulation, we assume that a diffractometer similar to the High Pressure Preferred Orientation (HIPPO) diffractometer is combined with the $\mathrm{D}-\mathrm{T}$ neutron source for diffraction experiments.[10] 
In order to estimate the resolution, MCNP is used to tally the neutrons with different energy bins and time bins. The resolution, $R$, is defined as:

$$
R=\frac{\delta_{d}}{d}
$$

where $\delta_{d}$ is the FWHM at $d$. The value of $d$ is calculated from Equation 2 with one major assumption: $t$ and $L$ are flight time and flight path length from the source to sample instead of from the source to the detector. The other assumption is that there is no angular divergence in the incident beam when the energy bins for each tally are calculated. In this case, an energy bin can be chosen, the width of which corresponds to the angular range covered by a single detector tube and the scattered angle for a given inter-atomic spacing, $d$.

The goal of the analysis below is to generate the diffraction spectrum for a chosen inter-atomic spacing. Such a spectrum allows one to estimate the resolution from the width of the peak in the distribution. In this method, we tally the neutrons of known energy in a time spectrum, which is then converted into diffraction spectrum to simulate the experimental angular and time-of-flight uncertainties. For this Monte Carlo based analysis, a prudent choice of energy and time bins is needed to keep the computation efficient (i.e. to minimize the computer time). In the analysis, one tally is setup for each angular interval representing a detector tube in experiment. The number of energy bins per tally is chosen according to the number of inter-atomic spacings of interest. The energy bins of each tally have slightly different width from each other because each detector tube in a detector panel corresponds to a slightly different scattered angle. 
The time bins are chosen so that each time bin of each tally corresponds to the same inter-atomic spacing, $d$. With the assumptions described in the previous paragraphs and a set of carefully picked time bins, $d$ can be estimated efficiently from the neutron intensity distribution. By superimposing the tallies converted to inter-atomic spacings, we can sum the number of counts of corresponding energy bins of each tally and construct a diffraction spectrum for the corresponding inter-atomic spacing (see Figures 5 to 9). The resolution, $R$, can be estimated from the FWHM of the peaks in the diffraction spectra.

The assumptions made in this scheme can be validated to be reasonable by the following error analysis. The length of flight path, $L$, is a known value with small uncertainties due to the angular divergence of the incident neutron beam, sample size, and the detector size. If these uncertainties of $L$ are neglected, the error of $d$ due to the error propagation of $\theta$ and $t$ derived from Bragg's Law can be written as:

$$
\left(\frac{\delta_{d}}{d}\right)^{2}=\left(\frac{\delta_{t}}{t}\right)^{2}+\left(\frac{\delta_{\theta}}{\tan \theta}\right)^{2}
$$

where $\delta_{t}$ is the uncertainty due to ToF and $\delta_{\theta}$ is the uncertainty due to beam divergence. A complete version of this equation is derived with the uncertainties of $L$ taken into account:[11]

$$
\left(\frac{\delta_{d}}{d}\right)^{2}=\left(\frac{\delta_{t}}{t}\right)^{2}+\left(\frac{\delta_{L}}{L}\right)^{2}+\left[\left(\frac{W_{\mathrm{det}}}{L_{2}}\right)^{2}+\left(\frac{W_{\text {sample }}}{L_{1}}\right)^{2}+\delta_{\theta}^{2}\right] \cdot \cot ^{2} \theta
$$

where $\delta_{L}$ is the uncertainty due to $\mathrm{L}, W_{\text {det }}$ is the diameter of the detector tube, and $W_{\text {sample }}$ is the width of the sample.

In Equation $5, W_{\text {sample }} / L_{1}$ is approximately the angular divergence of the neutron beam. The incident angle is determined by the collimator of the system. 
The divergence of the neutron beam is set to $0.32^{\circ}$ by a collimator. Because the geometry is treated exactly in MCNP up to the sample position, the uncertainty due to this angular divergence is taken into account. $W_{\text {det }} / L_{2}$ is approximately equal to the angular divergence due to the diameter of a single detector tube and is not taken into account in MCNP automatically. A typical diameter for a detector tube is $1.25 \mathrm{~cm}$. The flight path from sample to detector ranges from $0.75 \mathrm{~m}$ to $2 \mathrm{~m}$. A typical maximum sample size for HIPPO is $2 \mathrm{~cm}$ in diameter.[10] Therefore, $W_{\text {det }} / L_{2}$ is less than $0.05 \%$ which is negligible. The angular uncertainty, $\delta_{\theta}$, due to the finite detector size is included by tallying the neutrons into energy bins corresponding to the energy ranges covered by each detector.

Our MCNP model includes the source, moderators, collimators, and sample. Beyond the sample, because MCNP cannot handle diffraction scattering, the scheme described above is used to simplify the calculations. Detector panels usually have a plane geometry and are tilted with an angle so the exact length of the flight path from moderator to detector, $L$, is unknown. This tilted angle depends on the length of the detector tube and the scattered angle, $2 \theta$. These two factors change the sample to detector distance, $L_{2}$. In our scheme, a constant $L_{1}$ is used to calculate resolution, $R$, rather than $L$. Because $L_{1}$ is shorter than $L$ (i.e. $L_{1}+L_{2}$ ), the resolution calculated from $t$ at sample position as calculated with our scheme includes $\delta_{L}$ and gives a conservative estimation of $R$. 


\section{Simulation of a D-T neutron source}

The neutron source simulated here is an axial D-T neutron generator with two conical targets as schematically shown in Fig. 3. The multiple conical targets design has two advantageous features: a large target area and a minimal void space. The large target area allows the source to operate at a high beam current with sufficient cooling. The small void space in conical targets helps to reduce the neutron leakage from the neutron source without moderation. In our modeling, each conical target has a base diameter of $8 \mathrm{~cm}$ and a height of $8 \mathrm{~cm}$. The target consists of a thin (i $0.5 \mathrm{~mm}$ thick) titanium layer on an aluminum backing.

The conical targets are surrounded by a rectangular beryllium moderator with a dimension of $32 \mathrm{~cm}$ by $32 \mathrm{~cm}$ by $16 \mathrm{~cm}$. Beryllium is used to enhance the neutron yield because it has a large $(n, 2 n)$ reaction cross section. A polyethylene moderator next to the Be moderator with a surface area of $13 \mathrm{~cm}$ by $13 \mathrm{~cm}$ and a thickness of $4 \mathrm{~cm}$ is surrounded by a nickle reflector (see Fig. 4). The simulated collimation system is modeled after the collimation system for the HIPPO diffractometer at LANSCE in order to be able to compare the results.[10] The collimator system is $1 \mathrm{~m}$ away from the surface of the polyethylene moderator. It views a circular portion of the moderator surface with a diameter of $8.7 \mathrm{~cm}$ so that the neutron beam divergence is $0.32^{\circ}$. The first two meters of the collimator system consists of $1.5 \mathrm{~cm}$ thick iron plates. Each iron plate is separated by $0.5 \mathrm{~cm}$. The iron collimator system is followed by a $2.25 \mathrm{~m}$ thick tungsten collimator system which is made up of tungsten plates with a thickness and spacing in between similar to that of iron collimator system. Iron and tungsten are the shielding materials of choice because 
they have large neutron cross sections and high atomic numbers.

Fusion-based neutron generators need to be operated with relatively long ion pulse widths because they do not feature a beam bunching system. In contrast, a short pulse spallation neutron source (SPSS) can have a primary proton beam pulse width of $500 \mathrm{~ns}$, which is small compared to the neutron moderation time. This necessitates an optimization of flight path length, pulsing frequency, and ion beam pulse width in order to achieve desired neutron count rates and resolution. Based on these considerations we have chosen to position the sample six meters away from the surface of the polyethylene moderator. The flight path of our model is $3 \mathrm{~m}$ shorter than that of HIPPO diffractometer at LANSCE. However, both collimator systems have the same beam divergence. Although a shorter flight path allows a higher pulsing frequency, it also reduces the resolution of hot and thermal neutrons. When the source is operating at a high frequency, a time chopper can be incoporated to avoid frame overlap by blocking the higher energy neutrons. Our thermal neutron source with a flight path length of $6 \mathrm{~m}$ may operate at a frequency of $100 \mathrm{~Hz}$ when a time chopper is used to block the hot and fast neutrons. Based on a selected energy resolution for $0.025 \mathrm{eV}$ neutrons, a $50 \mu$ s ion beam pulse width is chosen for the $\mathrm{D}-\mathrm{T}$ neutron source in our simulation.

Table 2 shows two detector banks of the HIPPO diffractometer and their angular ranges which are used in the simulations. Each detector bank is capable of detecting scattered neutrons within a certain range of neutron energies. Table 2 also has a list of inter-atomic spacings chosen for the simulations. Our calculations have been restricted to two detector banks, at $20^{\circ}$ and at $150^{\circ}$ in order to limit computer time. For instance, the detector bank at $20^{\circ}$ has 32 detector tubes corresponding to 32 tallies. There are 32 matrices (each with 
2555 elements) stored in the physical memory for tallying the neutron signals at the $20^{\circ}$ detector bank. Therefore, the computer time increases significantly if two detector banks are calculated in one problem.

To reduce the computer time, a variance reduction technique, DXTRAN, has been used for the calculation.[12] A DXTRAN sphere can be defined to enclose a small region of interest when this region is inadequately sampled. Upon particle collision outside the DXTRAN sphere, a DXTRAN particle is created with the weight adjusted for the biased scattering angle. The DXTRAN particle is scattered to a randomly sampled position on the surface of the DXTRAN sphere. As a DXTRAN particle is transported deterministically without collision to the surface of the DXTRAN sphere, its weight is exponentially decreased by its optical path. The non-DXTRAN particle continues to be sampled in the normal way until it enters the DXTRAN sphere. When the non-DXTRAN particle enters the DXTRAN sphere, it is killed in order to balance the particle weight contributed to the cells inside the DXTRAN sphere. This technique is useful for our simulations because the sample in our problems is a region inadequately sampled. A DXTRAN sphere with a diameter of $2 \mathrm{~cm}$ is defined in our input files at the sample position to bias the scattering direction.

Our simulation shows that $50 \mu$ s is a reasonable choice for the ion beam pulse width of the $\mathrm{D}-\mathrm{T}$ neutron source. If the ion beam pulse width is longer than $50 \mu \mathrm{s}$, the resolution of thermal neutrons is more than $5 \%$. Figure 5 shows the resolution at $d=18.04 \AA$ and $2 \theta=20^{\circ}$. The energy covered by this detector bank for an inter-atomic spacing of $18.04 \AA$ is between 1.26 and 2.49 meV. This energy range corresponds to the cold neutrons. It has a resolution of $1.43 \%$. Figure 6 shows the resolution at $d=8.62 \AA$ and $2 \theta=20^{\circ}$. Its 
corresponding neutron energy range is 5.5 to $10.9 \mathrm{meV}$. This energy range falls in between cold and thermal energy. Its resolution is $1.58 \%$. As indicated by Equation 4, a better resolution can be achieved with lower energy (colder) neutron because their flight time is longer at the same scattered angle. This allows the use of longer beam pulse for cold neutron scattering.

The duration of a neutron beam pulse is a function of ion beam pulse width and the moderation time. Cold neutrons have a long moderation time so the cold neutron pulse obtained from one detector tube tends to overlape significantly with other cold neutron pulses obtained from other detector tubes on the same detector panel. On the contrary, thermal neutrons have a relatively shorter moderation time so that only thermal neutron pulses obtained from adjacent detector tubes tend to overlape each other. In addition, thermal neutrons have a much shorter flight time, so the primary ion beam pulse width has a much more significant effect on the resolution. Figure 7 shows the effects of ion beam pulse width for a hot neutron beam at $d=1.97 \AA$ and $2 \theta=20^{\circ}$. The resolution of hot neutrons improves by more than three times when the ion beam width is reduced from $50 \mu \mathrm{s}$ to $0 \mathrm{~s}$. The ion beam pulse width is relatively large when compared with the sum of flight time and slowing down time for hot neutrons. This result indicates that a short ion beam pulse width is favorable for a hot or thermal neutron source.

The $20^{\circ}$ detector bank covers 24.2 to $47.8 \mathrm{meV}$ at $d=4.12 \AA$ while the $150^{\circ}$ detector bank covers 37.1 to $39.3 \mathrm{meV}$ at $d=0.76 \AA$. Figure 8 shows the resolution at $d=4.12 \AA$ and $2 \theta=20^{\circ}$ while Fig. 9 shows the resolution at $d=0.76 \AA$ and $2 \theta=150^{\circ}$. As predicted by Equation 4 , the resolution for thermal neutrons is slightly larger than $3 \%$ with an ion beam width of $50 \mu \mathrm{s}$. Figures 8 and 9 also show that, as expected, the resolution at a large angle 
is slightly better than the resolution at a small angle. Unlike cold neutrons, thermal neutrons have a shorter moderation time and neutron flight time so the resolution is dominated by the energy resolution (i.e. $\left.\delta_{t} / t\right)$ instead of by the angular resolution. The total thermal neutron flux $\left(E_{\mathrm{n}}<1 \mathrm{eV}\right)$ at the sample position is $7.3 \times 10^{5} \mathrm{n} / \mathrm{cm}^{2} \mathrm{~s}$ assuming a neutron generator source strength of $10^{14} \mathrm{n} / \mathrm{s}$. The resolution is approximately $1 \%$ to $4 \%$ depending on the neutron energy and the scattered angle.

\section{Conclusions}

This study shows that a $\mathrm{D}-\mathrm{T}$ fusion-based neutron generator with a yield of $10^{14} \mathrm{n} / \mathrm{s}$ may produce a thermal neutron flux of $7.3 \times 10^{5} \mathrm{n} / \mathrm{cm}^{2} \mathrm{~s}$ at the sample position while providing a resolution between $1 \%$ and $4 \%$. The higher resolution achievable with cold neutrons makes the source particularly suitable for cold neutron scattering experiments. For a resolution comparable to the HIPPO diffractometer at LANSCE (i.e. $2.6 \%$ at $20^{\circ}$ ), the thermal neutron flux of the conceptual D-T neutron generator at the sample holder is only 13 times less than that of HIPPO with LANSCE operating at $70 \mu \mathrm{A}$.[10] It can be concluded that, when combined with a high efficiency diffractometer like HIPPO, such a D-T neutron generator has the potential of being a cost-

effective neutron source for neutron scattering experiments at universities and small research institutes. 


\section{Acknowledgements}

The authors wish to thank Prof. H.-R. Wenk for valuable discussion. This work has been supported by the U.S. Department of Energy under contract number DE-AC03-76SF00098.

\section{References}

[1] W.L. Bragg, X-Ray Crystallography, Sci. Am., 219, 58-70, (1968).

[2] T. Beas, Nuclear Research Reactors in the World, (1999).

URL http://www.iaea.org/worldatom/rrdb

[3] R. Pynn, Neutron Scattering: A Primer, Los Alamos Science, (1990).

[4] J. Reijonen, G. English, R. Firestone, et al, Compact Neutron Generator Development at LBNL, Accelerator Applications in a Nuclear Renaissance, ANS Annual Meeting in San Diego, (2003).

[5] I.I. Degtyarev, O.A. Liashenko, I.A. Yazynin, et al, Calculational Estimations of Neutron Yield from ADS Target, Proceedings of the 2001 Particle Accelerator Conference, Chicago, 2796-8, (2001).

[6] J.B. Donahue, T.O. Burn, P.D. Ferguson, et al, New Spallation Target and Moderator System at the Lujan Center, LANSCE Activity Report 1995-1998, 138-40, (1998).

[7] J.F. Briesmeister, ed., MCNP - A General Monte Carlo N-Particle Transport Code, LA-12625-M, Los Alamos National Laboratory, (2000).

[8] VITESS Module Source, Virtual Instrumentation (VITESS) for pulsed and continuous sources, (2001).

URL http://www.hmi.de/projects/ess/vitess/DOC/vitess.pdf 
[9] G.L. Squires, Introduction to the Theory of Thermal Neutron Scattering, Cambridge Univ. Press, (1978).

[10] K. Bennett, R.V. Dreele, H.-R. Wenk, HIPPO (High Pressure Preferred Orientation) Research Applications, 33, (1998).

[11] X.-L. Wang, Conceptual Design of the SNS Engineering Diffractometer, 12, (2000).

[12] T.E. Booth, A Sample Problem for Variance Reduction in MCNP, LA-10363MS, Los Alamos National Laboratory, (1985). 


\section{List of Figures}

1 A two-dimensional representation of Bragg's law 17

2 Relationship between the detector panel and sample position 18

3 Schematic diagram of the axial D-T neutron generator $\quad 19$

4 Geometry of thermal neutron scattering model in MCNP 20

5 Simulated diffraction spectrum for $d=18.04 \AA$ and $2 \theta=20^{\circ}$ $\begin{array}{ll}\text { (ion beam width }=50 \mu \mathrm{s}) & 21\end{array}$

6 Simulated diffraction spectrum for $d=8.62 \AA$ and $2 \theta=20^{\circ}$ (ion beam width $=50 \mu \mathrm{s}$ )

$7 \quad$ Resolution effect on hot neutrons due to primary ion beam width $\left(d=1.97 \AA\right.$ and $\left.2 \theta=20^{\circ}\right)$

8 Simulated diffraction spectrum for $d=4.12 \AA$ and $2 \theta=20^{\circ}$ (ion beam width $=50 \mu \mathrm{s}$ )

9 Simulated diffraction spectrum for $d=0.76 \AA$ and $2 \theta=150^{\circ}$ (ion beam width $=50 \mu \mathrm{s}$ ) 


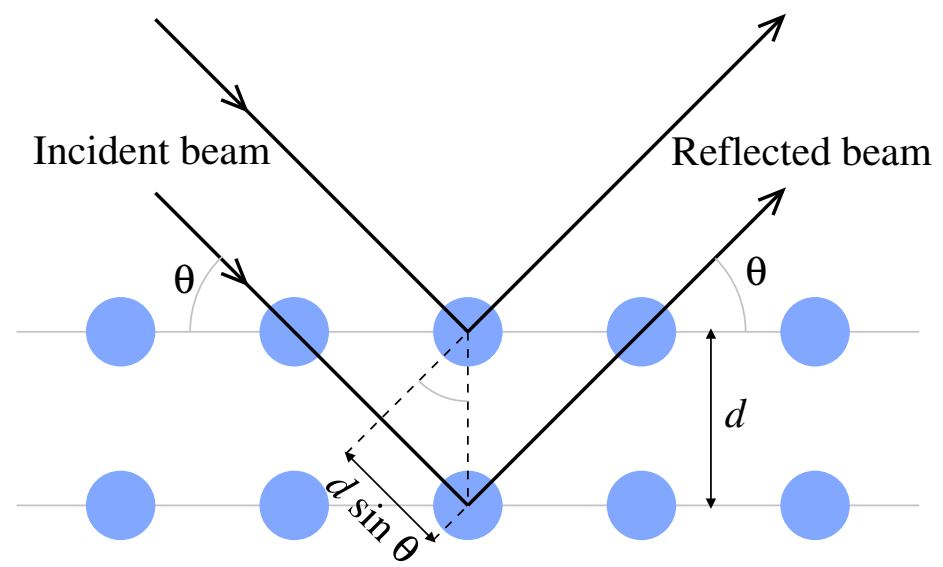

Fig. 1. A two-dimensional representation of Bragg's law 


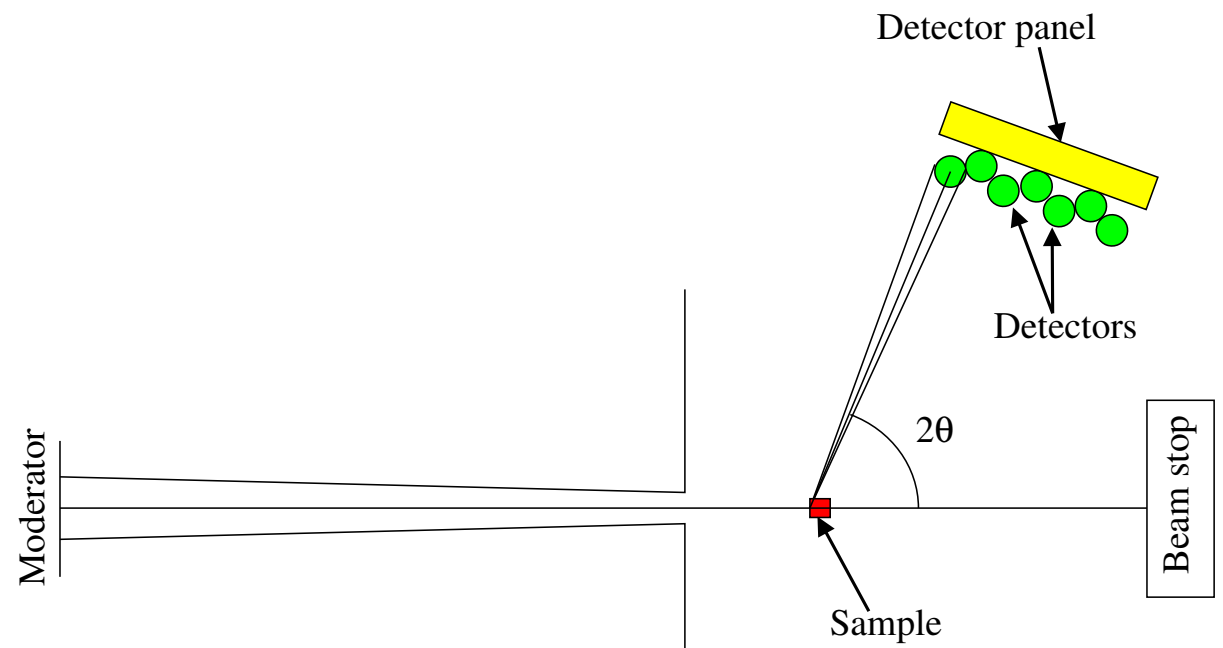

Fig. 2. Relationship between the detector panel and sample position 


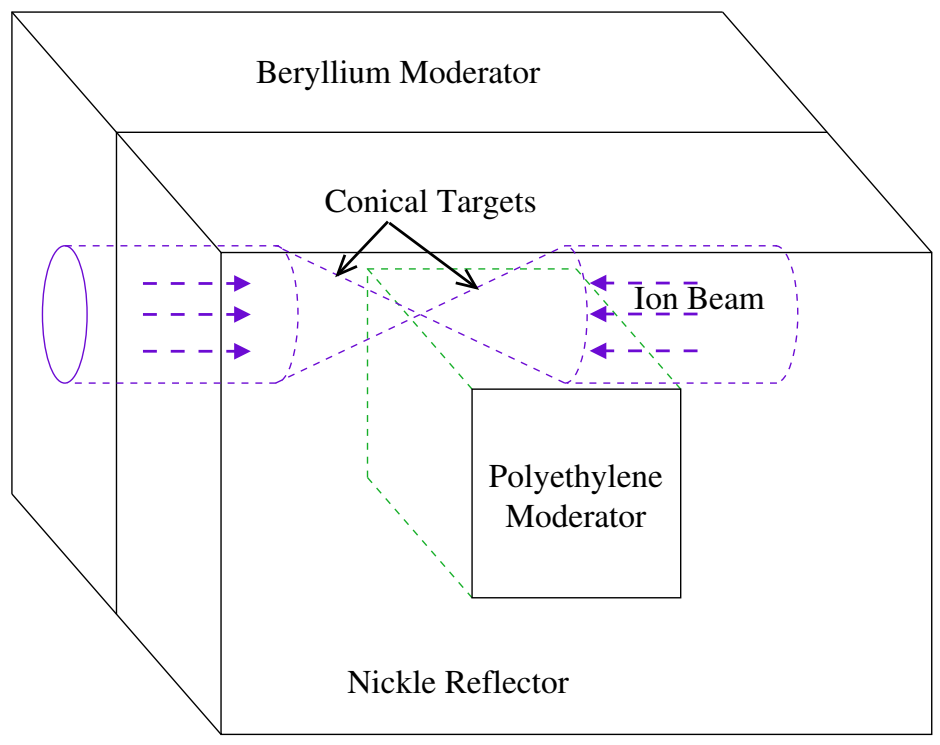

Fig. 3. Schematic diagram of the axial $\mathrm{D}-\mathrm{T}$ neutron generator 


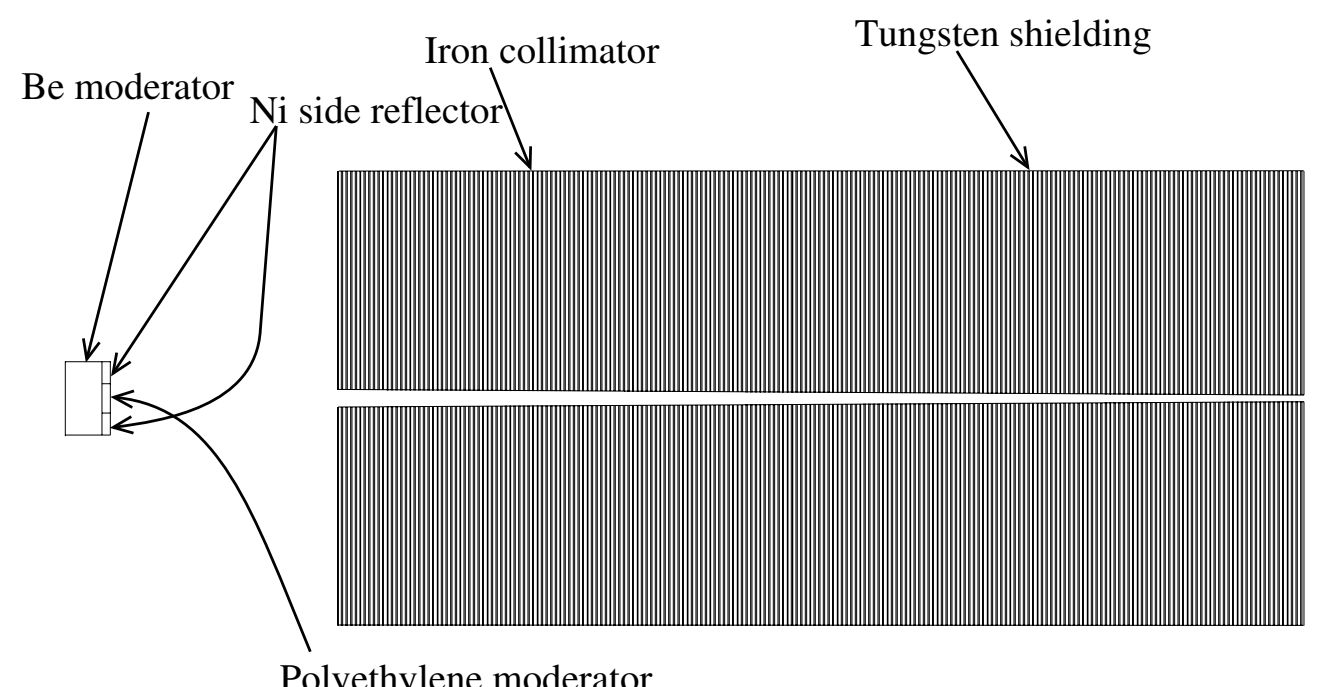

Polyethylene moderator

Fig. 4. Geometry of thermal neutron scattering model in MCNP 


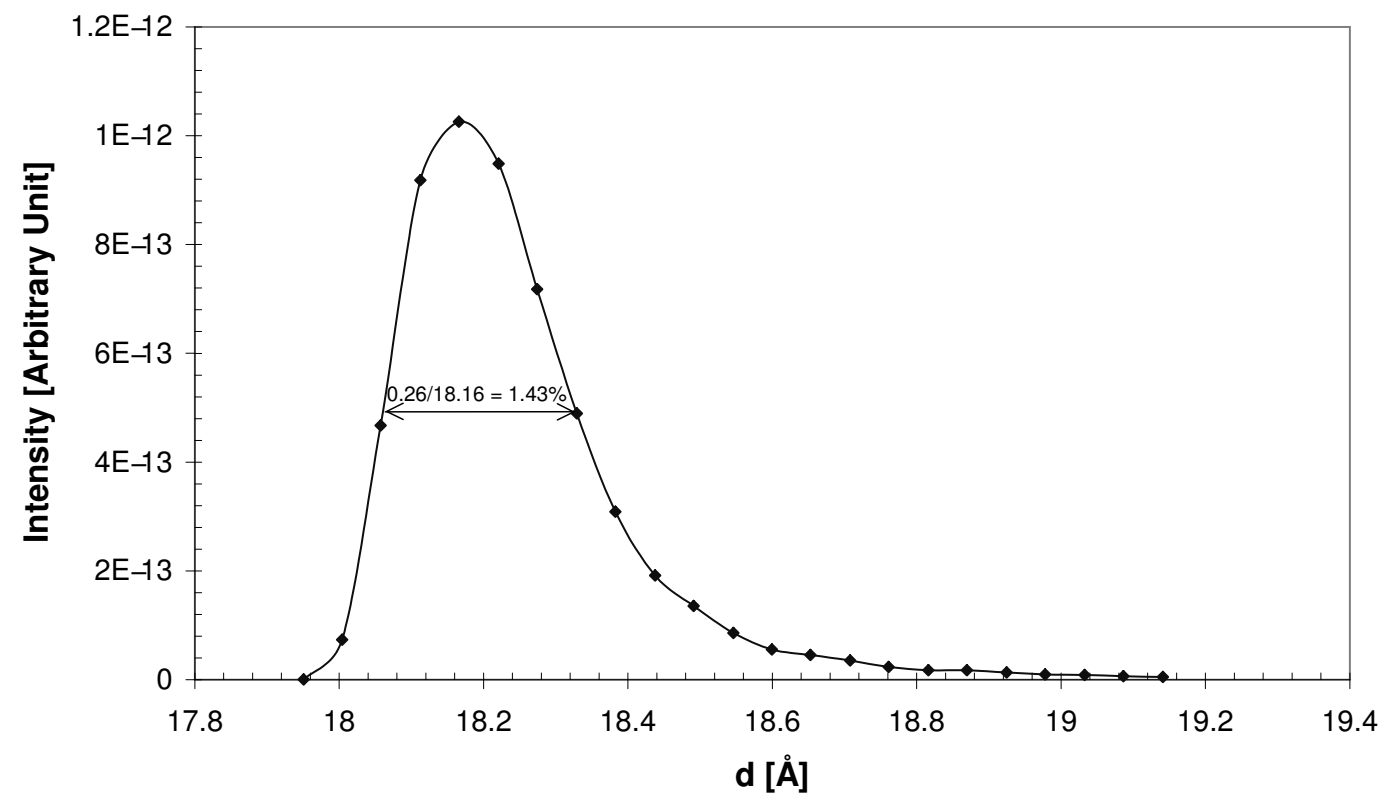

Fig. 5. Simulated diffraction spectrum for $d=18.04 \AA$ and $2 \theta=20^{\circ}$ (ion beam width $=50 \mu \mathrm{s})$ 


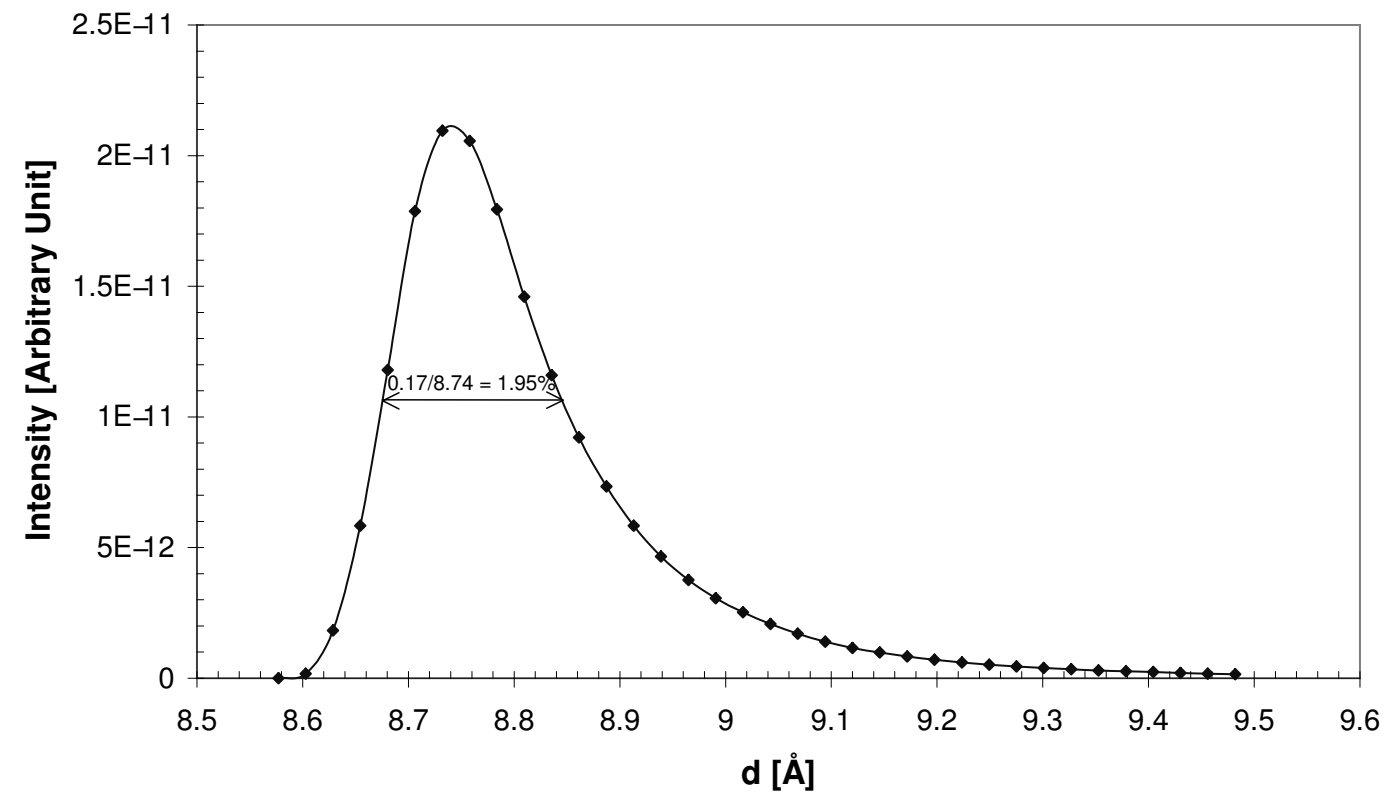

Fig. 6. Simulated diffraction spectrum for $d=8.62 \AA$ and $2 \theta=20^{\circ}$ (ion beam width $=50 \mu \mathrm{s})$ 


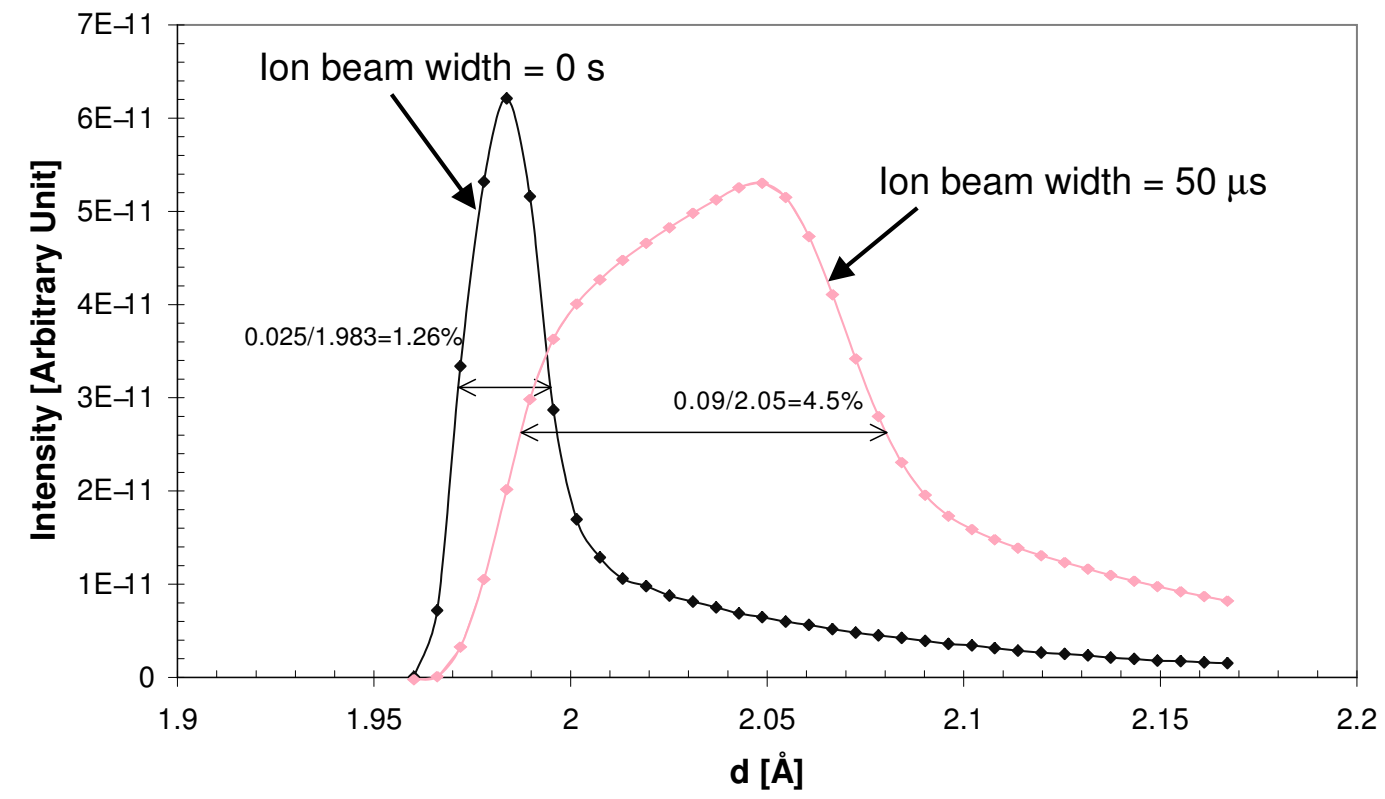

Fig. 7. Resolution effect on hot neutrons due to primary ion beam width $(d=1.97$ $\AA$ and $2 \theta=20^{\circ}$ ) 


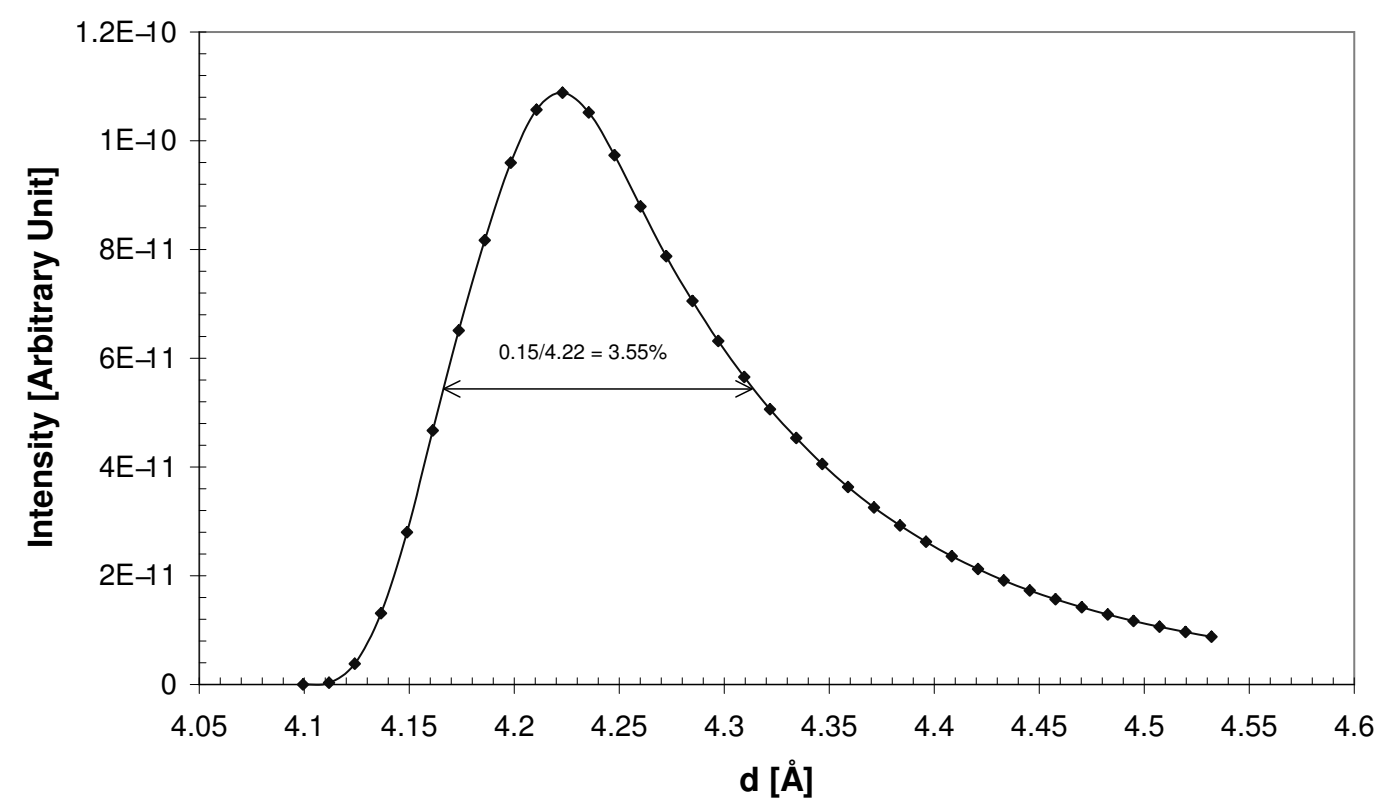

Fig. 8. Simulated diffraction spectrum for $d=4.12 \AA$ and $2 \theta=20^{\circ}$ (ion beam width $=50 \mu \mathrm{s})$ 


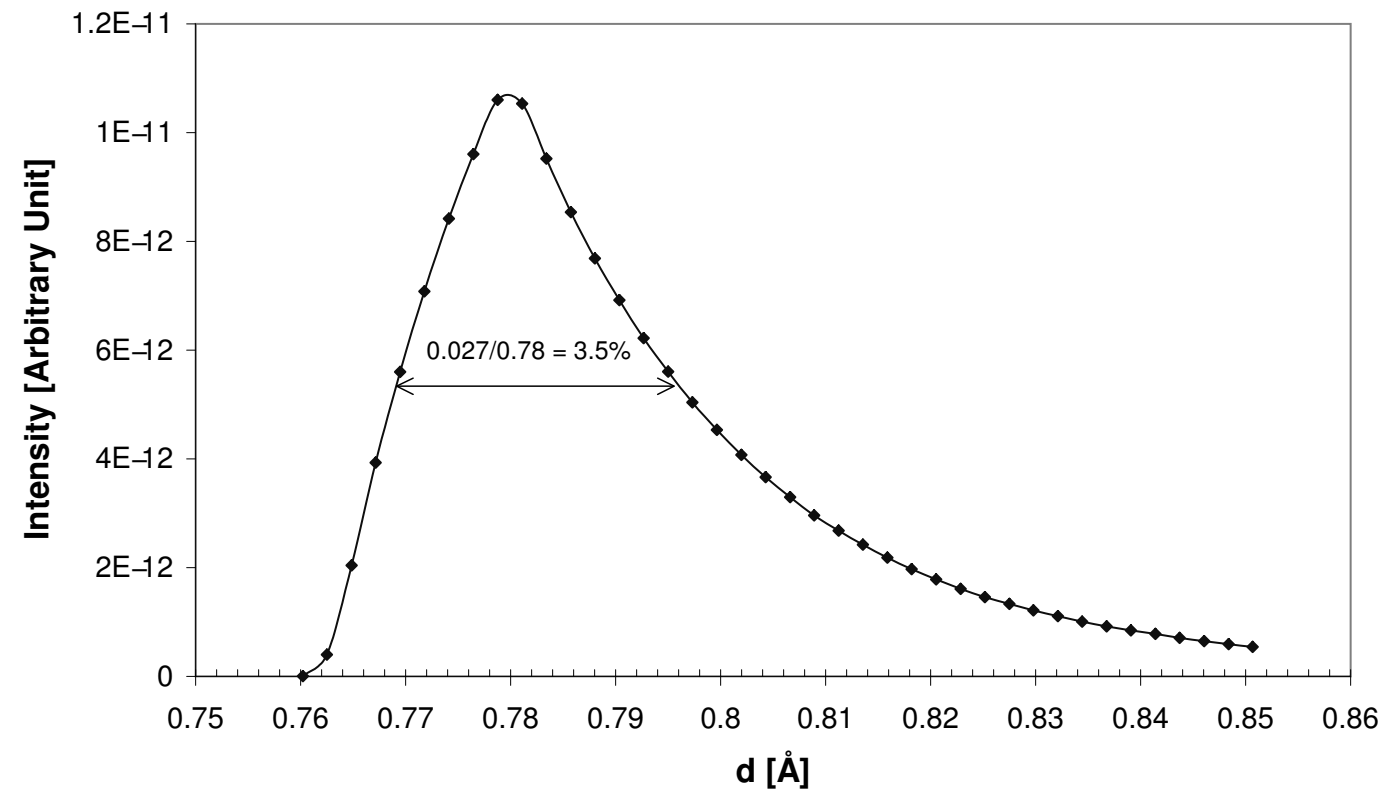

Fig. 9. Simulated diffraction spectrum for $d=0.76 \AA$ and $2 \theta=150^{\circ}$ (ion beam width $=50 \mu \mathrm{s})$ 
Table 1

Properties of neutrons for neutron scattering experiments $[9]$

\begin{tabular}{l|c|c|l|c}
\hline Source & Energy $(\mathrm{meV})$ & Temperature $\left({ }^{\circ} \mathrm{K}\right)$ & Wavelength $(\AA)$ & Velocity $(\mathrm{m} / \mathrm{s})$ \\
\hline cold & $0.1-10$ & $1-120$ & $30-3$ & $170-1700$ \\
thermal & $5-100$ & $60-1200$ & $4-1$ & $1200-5400$ \\
hot & $100-500$ & $1200-5800$ & $1-0.4$ & $5400-12000$ \\
\hline
\end{tabular}


Table 2: Neutron energies covered by certain detector banks of HIPPO for different atomic spacings

\begin{tabular}{c|c|c|c|c|c}
\hline Nominal angle & Angular range & $L_{2}(\mathrm{~m})$ & $d(\AA)$ & Nominal neutron energy $(\mathrm{meV})$ & Energy range $(\mathrm{meV})$ \\
\hline \multirow{3}{*}{$150^{\circ}$} & & & 0.36 & 166 & $162-172$ \\
& & & 0.52 & 80 & $77.7-82.1$ \\
& & & 0.76 & 38 & $37.1-39.3$ \\
& & \multirow{4}{*}{1.25} & 1.1 & 18.2 & $17.8-18.8$ \\
& & & 1.6 & 8.7 & $8.5-9.0$ \\
& & & 2.3 & 4.2 & $4.1-4.3$ \\
& & & 3.3 & 2 & $1.94-2.05$ \\
\hline \multirow{3}{*}{$20^{\circ}$} & & & 1.97 & 175 & $106-209$ \\
& & & 2.85 & 83.7 & $50.7-100$ \\
& & & 4.12 & 40 & $24.2-47.8$ \\
& & & 8.62 & 9.1 & $5.5-10.9$ \\
& & & 12.5 & 4.36 & $2.6-5.2$ \\
& & & 18.04 & 2.1 & $1.26-2.49$ \\
\hline
\end{tabular}

